\title{
Passive control of base pressure on an axisymmetric blunt body using a perimetric slit
}

\author{
Juan Marcos García de la Cruz, Anthony R. Oxlade, and Jonathan F. Morrison \\ Department of Aeronautics, Imperial College London, SW7 2AZ London, United Kingdom
}

(Received 7 April 2016; published 28 April 2017)

\begin{abstract}
The effect on the base pressure of a thin slit located at the base edge of a blunt axisymmetric body, communicating an internal cavity with the external flow, is investigated. A parametric study is performed of the effect on base pressure of changes in slit size and cavity depth. The base pressure increases initially with increasing cavity depth, but saturates at a depth which depends on the slit size. The base pressure increases monotonically up to $5 \%$ with increasing slit size for the geometries tested. An upper limit of base pressure recovery of $20 \%$ is extrapolated from the data. It is observed that the main effect of the slit is to reduce the instantaneous pressure asymmetry, which is linked to the total base pressure in a similar fashion for all the slit sizes. As a second-order effect, for highly asymmetric pressure distributions, the slit produces a base pressure increase not associated with the base pressure asymmetry. The results suggest a global effect of the slit on the wake due to a diametrical flow within the cavity driven by the pressure differences across the slit and regulated by the largest of the pressure drops between the slit and cavity. The slit also reduces the periodic base pressure fluctuations, corresponding mainly to the vortex shedding, and increases the rotational speed of the wake.
\end{abstract}

DOI: 10.1103/PhysRevFluids.2.043905

\section{INTRODUCTION}

The drag force acting on a body is equal to the energy per unit distance extracted from the approaching free stream by the body. For bluff bodies the drag force comprises primarily the axial pressure difference, with a relatively small contribution from skin friction. In turbulent flows, energy is extracted by both steady viscous stress gradients and production of turbulent motions which extract additional kinetic energy from the mean flow. It is well known that the turbulent wake typically comprises both incoherent motions and spatially organized or coherent structures, which are generally accepted to be a consequence of inherent laminar flow instabilities [1-6]. While the exact contribution of coherent structures to the drag remains an important unanswered question [3], their perceived significance is highlighted by the numerous studies which target large-scale vortex shedding as a strategy for reducing bluff-body drag. A recent review is provided by [7]. For two-dimensional (2D) bodies, suppression of vortex shedding is a particularly effective method of drag reduction, yielding base pressure recoveries of up to $60 \%$. This has been performed using a variety of techniques such as splitter plates [8,9], base bleed [10], or periodic blowing [11].

In the case of axisymmetric turbulent wakes, suppression of vortex shedding has proven inherently more difficult due to the three-dimensionality of the wake structure. Early studies $[1,2,4,5,12]$ identified the most energetic coherent structures in the far wake as antisymmetric, with azimuthal wave number $|m|=1$ and frequency $0.13 \lesssim \mathrm{St} \lesssim 0.27$ (dependent upon the body aspect ratio and Reynolds number). However, in addition to antisymmetric vortex shedding (a characteristic shared with $2 \mathrm{D}$ bodies) the wake also exhibits a random azimuthal global rotation. This was first observed in flow visualizations in [13], and subsequently characterized in terms of base pressure measurements in [14], which provided evidence that the wake barycenter exhibited a slow, random orbit around the body center with a nonzero mean radius. Further insight was provided by Rigas et al., [6], who showed that in a rotating frame of reference, the base pressure retains the symmetry-breaking properties (both steady and unsteady) of the laminar wake $[15,16]$ ). These analyses have recently been extended to include the velocity field by Gentile et al. [17], who shed light on the spatial 
variation of the wake structure: very close to the base, fluctuation energy is dominated by the slow rotation of the wake. Further downstream, the fluctuation energy becomes increasingly associated with vortex shedding. Therefore in contrast to $2 \mathrm{D}$ bodies, vortex shedding in the axisymmetric wake occurs off-center and at a randomly varying azimuthal angle. Such off-center oscillation, restricted to one dimension, can also be found in rectilinear bluff bodies [18-20]. The random rotation of the global wake structure (and angle of vortex shedding) on a very long time scale recovers the time-average axisymmetry. For a thorough characterization of the baseline wake in the current investigation, the reader is referred to $[6,14]$.

Compared with 2D bodies, wake control strategies for the axisymmetric blunt body are far less common and to our knowledge only four techniques have demonstrated successful control. In terms of active strategies, both base bleed [21] and periodic blowing [22] have been employed. The former targets vortex shedding in a manner similar to the 2D wake: pressure recovery is achieved by progressive inhibition and eventual suppression of antisymmetric vortex shedding at high bleed coefficients [23]. In contrast, periodic blowing [22] was shown to inhibit turbulent entrainment via a broadband suppression of base pressure fluctuations, without targeting any specific modes. Passive control methods [24,25] demonstrate suppression of vortex shedding by introducing external cavities into the wake; an early review is provided in [26]. More recently, researchers [27] showed that a cavity formed by extending the perimeter of the body base in the streamwise direction can increase the stability of the laminar wake and increase the base pressure. Although detailed characterization has yet to be extended to high Reynolds numbers, at transitional Reynolds numbers $\left(\operatorname{Re}_{D}<700\right)$ the energy of velocity fluctuations associated with the vortex-shedding mode are suppressed. Pressure drag reductions of up to $15 \%$ have been observed for cavity lengths greater than half the body diameter. In the present investigation we demonstrate a method for reducing drag by increasing the instantaneous symmetry of the wake which reduces the pressure fluctuations associated with antisymmetric vortex shedding, but does so with minimal modification to the external body geometry.

The rest of this paper is outlined as follows. The experimental setup is detailed in Sec. II. The results are presented and discussed in Sec. III before conclusions are made in Sec. IV.

\section{APPARATUS AND EXPERIMENTAL PROCEDURE}

Experiments were performed in a closed-circuit wind tunnel with a working section of size $1.37 \times 1.21 \times 3.0 \mathrm{~m}^{3}$, a contraction ratio of 4.92 and a free-stream turbulence intensity smaller than $0.1 \%$ at $15 \mathrm{~ms}^{-1}$. A proportional-integral-derivative (PID) algorithm controlled the free-stream velocity, $U_{\infty}=15 \mathrm{~ms}^{-1} \pm 0.2 \%$.

The axisymmetric bluff body with blunt trailing edge employed in the experiments, sketched in Fig. 1(a), has a body diameter $D=196.5 \mathrm{~mm}$ and total length $L=6.48 D$. The body nose describes a super-ellipse profile [28] with an aspect ratio of 2.5. Two strips of 120 grit emery paper of widths 2 and $25 \mathrm{~mm}$, respectively located at $z / L=0.884$ and $z / L=0.784$, conditioned the body boundary layer so as to match the typical geometrical proportion of full-size real road vehicles. The boundary-layer momentum thickness at separation was $\theta=2.14 \mathrm{~mm}$. The Reynolds numbers based on $D$ and $\theta$ are $\operatorname{Re}_{D}=1.88 \times 10^{5}$ and $\operatorname{Re}_{\theta}=2050$, which confirm that the boundary layer at separation is turbulent. A strut with a cross section of a blended NACA 0015/0030 aerofoil, and a constant thickness of $11 \mathrm{~mm}$ along its length, supported the model in the wind tunnel. Further details of the experimental setup are available in [14].

The rear end of the body contains a void cylindrical cavity with an internal diameter of $0.7 \mathrm{D}$, and a volume that can be varied by modifying the cavity depth $h$. The cavity is connected to the external flow through a perimetrical slit in the body base. The width of the slit $d$ is varied from $0 \mathrm{~mm}$ (baseline) to $5 \mathrm{~mm}$ in steps of $1 \mathrm{~mm}$. These values correspond to $2.5 \% \mathrm{D}$ and $0.5 \% \mathrm{D}$ and are respectively 2.34 and 0.47 times the value of $\theta$ at separation. The internal cavity depth $h$ is varied from $h / D=1.2 \%$ to $h / D=9.6 \%$.

A uniform polar grid with $\Delta r=11 \mathrm{~mm}=0.056 D$ and $\Delta \phi=45^{\circ}$ of 64 taps, as sketched in Fig. 1(b), was employed to measure the pressure distribution on the base of the model. The 
(a)

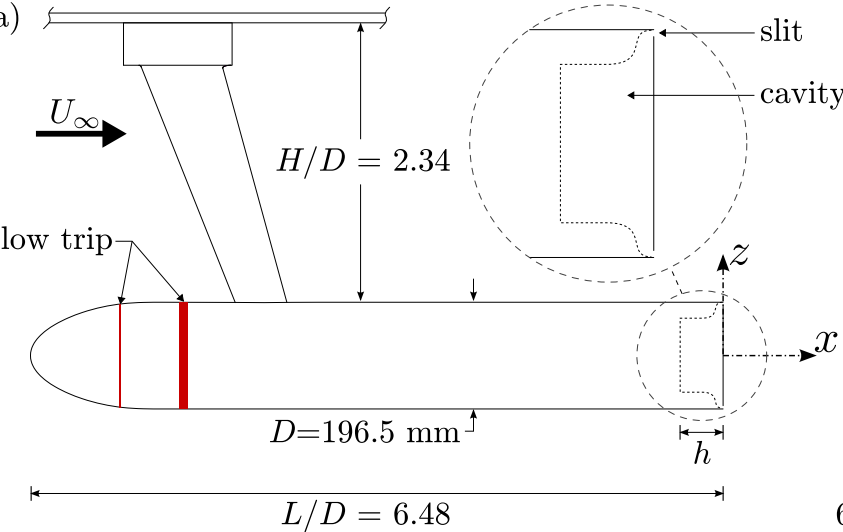

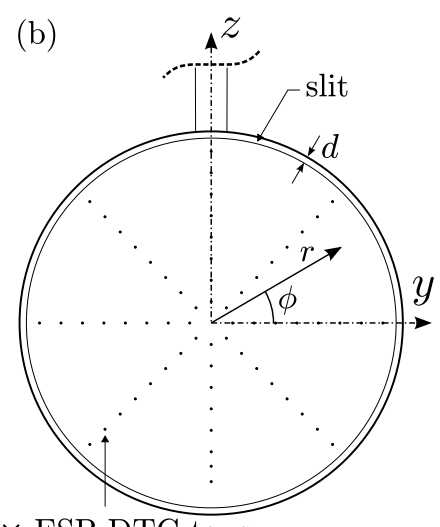

$64 \times$ ESP DTC taps

FIG. 1. Experimental setup. (a) Lateral view and detail of the rear cavity and slit and (b) rear view of the axisymmetric body employed in the experiments.

static pressure is measured using an ESP-64HD digital temperature-compensated (DTC) pressure scanner and a Chell CANdaq 14 bit analog-to-digital (A/D) converter with a sampling frequency of $225 \mathrm{~Hz}$. The static taps and tubing were calibrated for attenuation and phase shift against a Meggitt piezoresistive pressure transducer model $8507 \mathrm{C}-1$, with a resonant frequency of $55 \mathrm{kHz}$. The pressure coefficient $C_{p}(r, \phi, t)$ at a radius $r$ and an angle $\phi$ at time $t$ is calculated from the pressure signal $p(r, \phi, t)$ and the tunnel static pressure $p_{\infty}$ as

$$
C_{p}(r, \phi, t)=\frac{p(r, \phi, t)-p_{\infty}}{\frac{1}{2} \rho U_{\infty}^{2}},
$$

where $\rho$ is the air density in the tunnel. A modal azimuthal description of the pressure field is obtained by expanding $C_{p}$ into the $|m| \leqslant 4$ Fourier azimuthal modes $C_{p}^{m}(r, t)$ as

$$
C_{p}^{m}(r, t)=\frac{1}{2 \pi} \int_{0}^{2 \pi} C_{p}(r, \phi, t) e^{-2 \pi i m \phi} d \phi
$$

for which the spatial averages are

$$
\left\langle C_{p}^{m}(t)\right\rangle=\frac{8}{D^{2}} \int_{0}^{R} C_{p}^{m}(r, t) r d r
$$

The moduli and arguments of these modes are respectively represented as $\left|\left\langle C_{p}^{m}(t)\right\rangle\right|$ and $\angle\left\langle C_{p}^{m}(t)\right\rangle$. The temporal average of the pressure fields during a period $T$ is presented in a rotating frame of reference by averaging an angular translation of each field of $\angle\left\langle C_{p}^{1}\right\rangle$ :

$$
\overline{C_{p} \mid \angle\left\langle C_{p}^{1}\right\rangle}(r, \phi)=\frac{1}{T} \int_{0}^{T} C_{p}\left(r, \phi-\angle\left\langle C_{p}^{1}\right\rangle, t\right) d t .
$$

The spatial average base pressure $\left\langle C_{p}(t)\right\rangle$ in the area $A=\pi(D / 2)^{2}$ is obtained as

$$
\left\langle C_{p}(t)\right\rangle=\frac{1}{A} \int_{A} C_{p}(r, \phi, t) d A,
$$

for which $d A=r d \phi d r$. The spatiotemporal base-average pressure in a period $T$ is

$$
\left\langle\overline{C_{p}}\right\rangle=\frac{1}{T} \int_{0}^{T}\left\langle C_{p}(t)\right\rangle d t,
$$



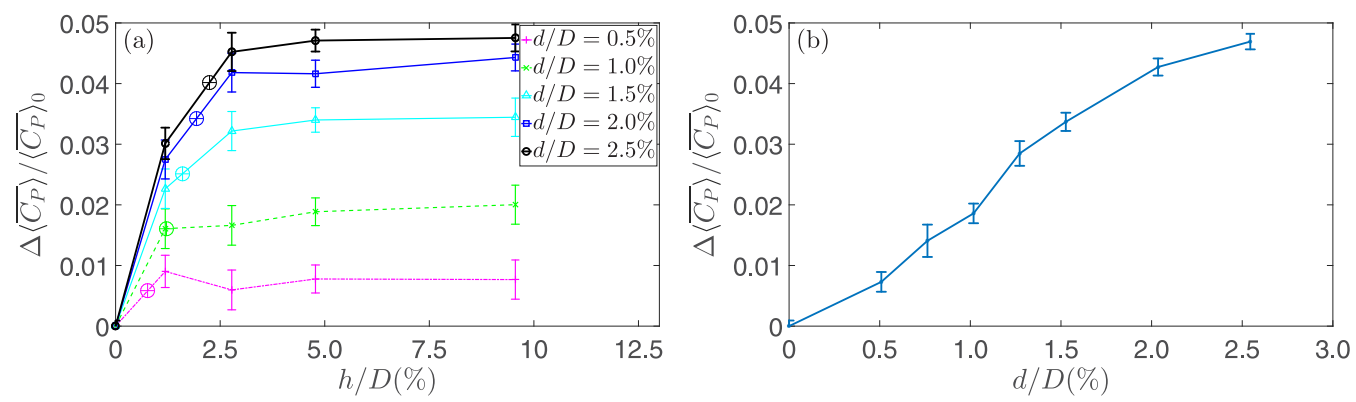

FIG. 2. Average pressure increment. Change in the pressure coefficient spatiotemporal averages with (a) slit size and cavity volume and (b) only slit size. In (a), $\oplus$ is located at $4 h_{s}^{3} \sim \mathcal{F} D d^{2}$ for $\mathcal{F}=0.07$.

and the total variance is

$$
\left\langle\overline{C_{p}^{\prime 2}}\right\rangle=\frac{1}{A T} \int_{A} \int_{0}^{T}\left(C_{p}-\overline{C_{p}}\right)^{2} d t d A .
$$

Temporal pressure fluctuations are presented in the frequency domain by projecting the absolute value and phase of the azimuthal modes onto Fourier modes:

$$
\begin{aligned}
& \left|\left\langle\tilde{C}_{p}^{m}\right\rangle\right|\left(\mathrm{St}_{D}\right)=\frac{1}{T} \int_{0}^{T}\left|\left\langle C_{p}^{m}(t)\right\rangle\right| e^{-2 \pi i U_{\infty} / D \mathrm{St}_{D} t} d t, \\
& \angle\left\langle\tilde{C}_{p}^{m}\right\rangle\left(\mathrm{St}_{D}\right)=\frac{1}{T} \int_{0}^{T} \angle\left\langle C_{p}^{m}(t)\right\rangle e^{-2 \pi i U_{\infty} / D \mathrm{St}_{D} t} d t,
\end{aligned}
$$

where $\mathrm{St}_{D}$ is the frequency $f$ normalized with $U_{\infty} / D$. The symbol $\Delta$ refers to the increment in pressure with respect to the baseline geometry (that without a slit), denoted by the subscript 0 . The error bars presented are for a $95 \%$ confidence.

\section{RESULTS}

The change in the spatiotemporal base-average pressure coefficient relative to the baseline, $\Delta\left\langle\overline{C_{p}}\right\rangle /\left\langle\overline{C_{p}}\right\rangle_{0}$, is shown in Fig. 2(a) for a range of cavity depths and slit sizes. For any slit size, $\left\langle\overline{C_{p}}\right\rangle$ increases with $h / D$ up to a saturation depth, $h_{s}$, after which further cavity enlargements of up to $244 \%$ do not lead to any noticeable pressure change. As observed, $h_{s}$ increases with $d$ : for the smallest slit, $h_{s}$ occurs at a smaller depth than the smallest tested, $h / D=1.2 \%$, while the pressure recovery of the largest slit saturates at $1.2 \%<h_{s} / D<2.8 \%$.

The dependence of $h_{s}$ on $d$ is compatible with the base pressure recovery relying on diametrical flow across the cavity. Such behavior can be modeled by a simplified geometry consisting on a 2D channel of length $D$ and height $h \ll D$, taking fluid and discharging it through two slits of size $d$. In such a geometry the flow would suffer a sudden expansion pressure drop when crossing each slit, $\Delta p_{s} \sim \frac{1}{2} \rho u_{s}^{2}$, and another pressure drop when crossing the cavity $\Delta p_{c} \sim \mathcal{F} \frac{D}{2 h} \frac{1}{2} \rho u_{c}^{2}$; where $v_{s}$ and $v_{c}$ are the velocities in the slit and cavity, and $\mathcal{F}$ is the friction factor in the channel from the Moody chart [29]. Making $\Delta p_{c}=2 \Delta p_{s}$ the condition for saturation of $h$ and, by continuity, $v_{s}=v_{c} h / d$, leads to $4 h_{s}^{3} \sim \mathcal{F} D d^{2}$, which exposes the positive correlation between $h_{s}$ and $d$. To frame this model into the data acquired, $h_{s}$ for $\mathcal{F} \sim 0.07$ (a large $\mathcal{F}$ is expected since the sensing tubing is exposed to the cavity) is presented in Fig. 2(a). Further analysis reveals that for all the slits, the rest of the statistics are equally independent of $h$ for $h / D \geqslant 2.8 \%$. As a consequence, to reduce statistical error, the results presented hereafter are the average for $h / D \geqslant 2.8 \%$.

The variation of the spatiotemporal averaged pressure coefficient increment, $\Delta\left\langle\overline{C_{p}}\right\rangle /\left\langle\overline{C_{p}}\right\rangle_{0}$, with slit size is shown in Fig. 2(b). This graph illustrates that the monotonic base pressure increase 

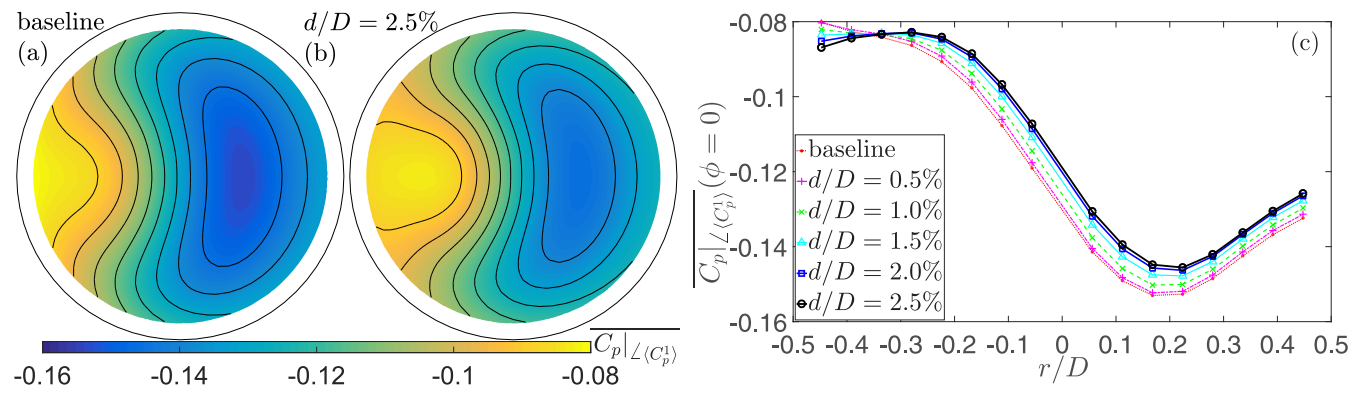

FIG. 3. Pressure temporal conditional average for (a) baseline and (b) $d / D=2.5 \%$ and for (c) all the slits at $\phi=0$. This is equivalent to a temporal average in a frame of reference rotating as $\angle\left\langle C_{p}^{1}\right\rangle$.

with slit size decreases its slope as $d$ increases. A base pressure increase of almost $5 \%$ is shown.

As noticed in [6], the instantaneous pressure field in the turbulent wake of an axisymmetric body contains the spatial asymmetry characteristic of laminar unsteady regimes. Such asymmetric flow is neutrally stable in the range $0<\phi<2 \pi$ and external disturbances distribute the wake azimuthal position uniformly, making the pressure field axisymmetric in the long time average [30]. The spatial distribution of the conditional temporal average of $C_{p}, \overline{\left.C_{p}\right|_{\angle\left\langle C_{p}^{1}\right\rangle}}(r, \phi)$, is presented in Fig. 3(a) for the baseline and in Fig. 3(b) for $d / D=2.5 \%$. Figure 3(c) presents the same average at $\phi=0$ for the slit sizes tested. The distributions presented provide insight into the instantaneous base pressure and the effect of the slit on the pressure field.

For all the configurations there is a pronounced asymmetry captured by the conditional average. As observed, the slit reduces $C_{p}$ locally just around the area of the highest pressure and increases $C_{p}$ in a similar proportion in the rest of the base, resulting in a net spatial average base pressure increase. The compound result of these two effects is a reduction in the pressure field asymmetry, often associated with a lateral and a drag force.

The effect that the slit has on the wake dynamics can be inferred from the changes that it produces in the base pressure fluctuations. Figure 4(a) presents the relation between slit size and the base average of the $C_{p}$ temporal variance, where a monotonic reduction of the base pressure fluctuations with slit size is observed. A detailed analysis of the base pressure fluctuations is performed by projecting the pressure fields onto orthogonal azimuthal modes and analyzing the variance content at different frequencies of the spatially averaged absolute value $\left|\left\langle\tilde{C}_{p}^{m}\right\rangle\right|$ and phase $\left\langle\left\langle\tilde{C}_{p}^{m}\right\rangle\right.$, as described by Eqs. (8) and (9). The frequency distribution of variance in the absolute value of the azimuthal wave numbers $m=0$ to $m=2$ are presented in Figs. 4(b) and 4(c). The frequency distribution of variance in the (unwrapped) phase of the azimuthal wave numbers $m=1$ and $m=2$ are presented in Fig. 4(d). All spectra are presented for the baseline, $d / D=1.0 \%$ and $d / D=2.5 \%$. The variance content on modes $m=3$ and $m=4$ were both small and barely altered by the slit size, so they are omitted.

As observed, all the spectra contain a nearly -2 slope power law at high $\mathrm{St}_{D}$, characteristic of random Brownian dynamics [30]. The pressure fluctuations can be split into "drumming" (phase independent of time) oscillations and azimuthal rotation, the former being captured by $\left|\left\langle\tilde{C}_{p}^{m}\right\rangle\right|$ in Figs. 4(b) and 4(c) and the latter by $\angle\left\langle\tilde{C}_{p}^{m}\right\rangle$, in Fig. 4(d). Drumming fluctuations occur down to a minimum frequency, constrained by the body geometry and the flow regime, leading to a saturation of the variance content at $\mathrm{St}_{D}<5 \times 10^{-2}$ in Figs. 4(b) and 4(c). On the contrary, as presented in Fig. 4(d), the random azimuthal rotation is not bounded and the azimuthal motion presents an unlimited Brownian diffusion [30]. Because modes are statistically in phase, their angular motion is faster for higher modes, whose wave numbers perceive a larger phase shift for the same angle, as evidenced by the shift between $m=1,2$ in Fig. 4(d). 

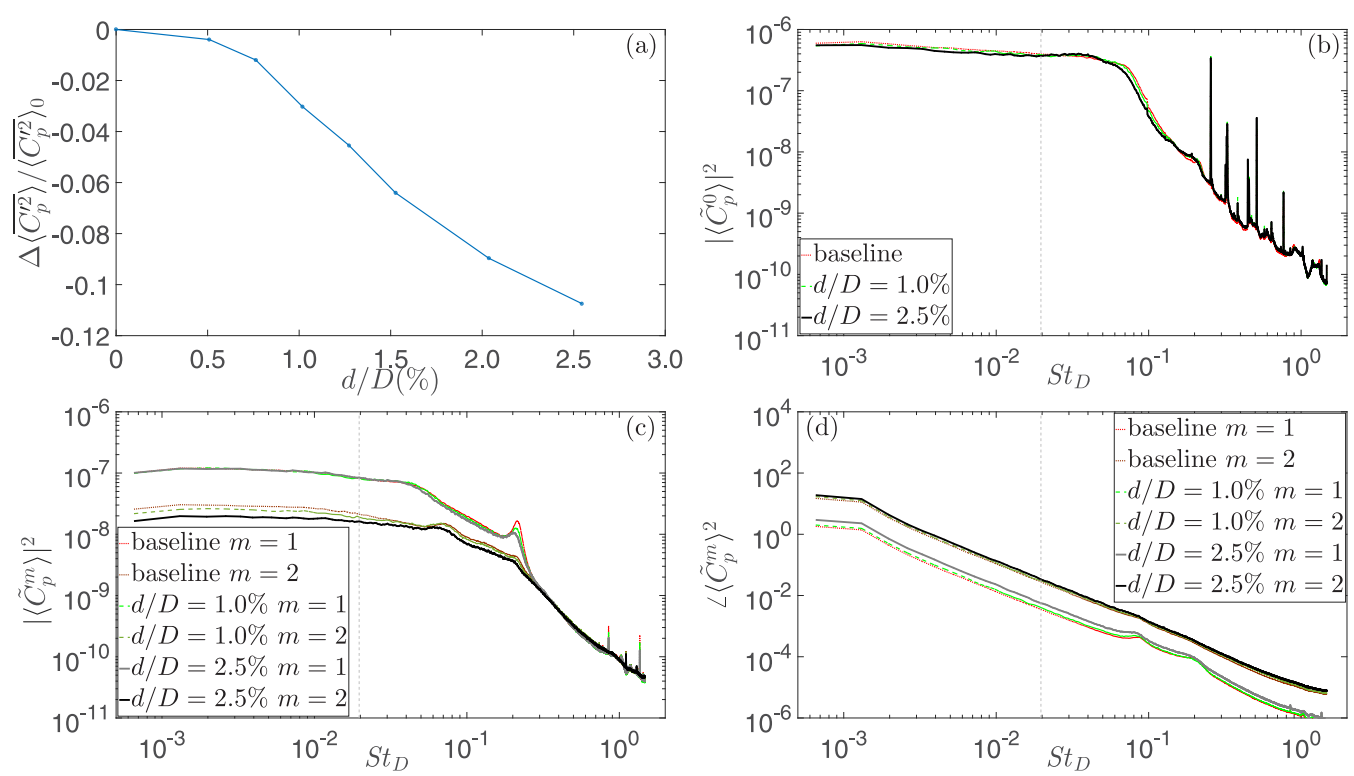

FIG. 4. Pressure fluctuations. (a) Variation of average pressure fluctuations with slit size. Spectral content of variance in the absolute value of modes (b) 0 , (c) 1 and 2, and (d) in the phase of modes 1 and 2.

The two periodic modes present in the pressure variance spectra are the vortex shedding at $\mathrm{St}_{D} \sim 2 \times 10^{-1}$, and what is believed to be its subharmonic [31] at $S t_{D} \sim 8 \times 10^{-2}$. Vortex shedding is clearly recognizable in $\left|\left\langle\tilde{C}_{p}^{1}\right\rangle\right|$, and slightly affects $\left|\left\langle\tilde{C}_{p}^{2}\right\rangle\right|$ or $\angle\left\langle\tilde{C}_{p}^{1}\right\rangle$. It can be concluded that this periodic mode is mostly a drumming phenomenon with an azimuthal periodicity of 1 , even if some of its energy is leaked toward higher wave numbers and the rotating wake motion. As shown in Fig. 4(c), the slit produces a reduction in this periodic oscillation: pressure fluctuations are reduced by $70 \%$ in $\left|\left\langle\tilde{C}_{p}^{1}\right\rangle\right|$ at $\mathrm{St}_{D} \sim 2 \times 10^{-1}$ from baseline to $d / D=2.5 \%$.

It can be observed in Fig. 4(d) how, in spite of reducing the average pressure fluctuations, the slit increases the azimuthal oscillation of the wake across the entire frequency spectrum. This is the result of a more symmetric wake: in a stochastic weakly nonlinear model of the wake dynamics [30], random perturbations would make a more symmetric wake rotate faster, increasing the azimuthal position variance. A similar effect is observed in the symmetrization of Ahmed body wakes [32].

The reduction in the pressure field asymmetry, observed in Fig. 3 and behind the increase in the speed of the wake rotation presented in Fig. 4(d), can also be related to the effectiveness of the slit: as hypothesized before, it is the pressure imbalance around the slit that produces the flow across the internal cavity. To account for such a pressure imbalance, asymmetry $\mathcal{A}$ is defined as the spatial standard deviation of $C_{p}$ in the outermost radius,

$$
\mathcal{A}(t)=\sqrt{\sum_{m \neq 0} C_{p}^{m}\left(r_{\max }, t\right)^{2}},
$$

and correlated with the base pressure spatial average $\left\langle C_{p}\right\rangle(t)$. The coherence between these two functions (not plotted) is low for $\mathrm{St}_{D} \gtrsim 10^{-2}$ but reaches a plateau of about 0.6 for $\mathrm{St}_{D} \lesssim 2 \times 10^{-2}$, about ten vortex-shedding cycles. Under this threshold, marked in Fig. 4(b)-4(d), the random fluctuations, caused by the modal Brownian dynamics, that reduce the coherence between the signals are restrained by the body geometry, increasing the coherence between $\mathcal{A}$ and $\left\langle C_{p}\right\rangle$.

Figure 5(a) presents the joint probability distribution between $\mathcal{A}$ and $\left\langle C_{p}\right\rangle$ for the baseline and $d / D=2.5 \%$ after applying a temporal moving average of size $t U_{\infty} / D=50$ (equivalent to $\mathrm{St}_{D}=0.02$ ). This moving average filters the random fluctuations that reduce the correlation between 

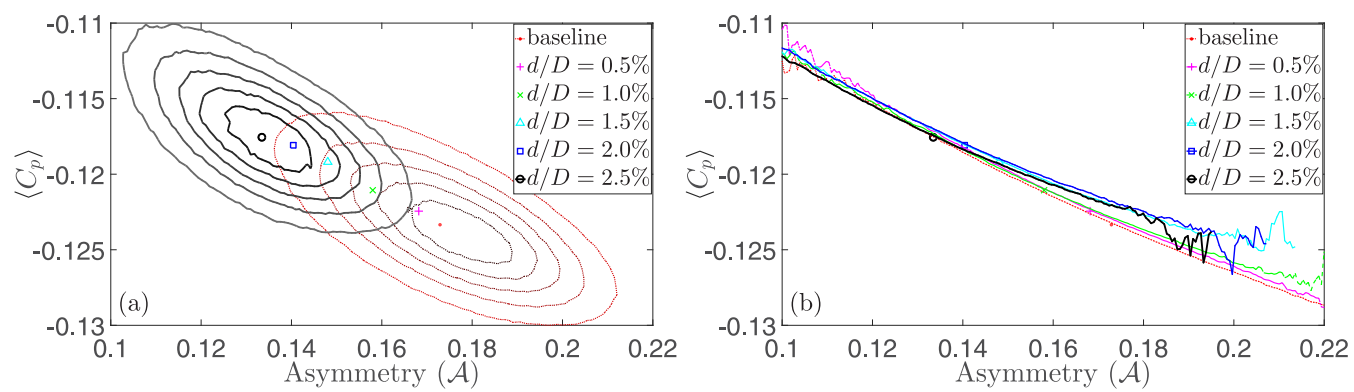

FIG. 5. Effect of asymmetry in base pressure. (a) Contour maps of the joint probability distribution between $\mathcal{A}$ and $\left\langle C_{p}\right\rangle$. (b) Conditional average of $\left\langle C_{p}\right\rangle$ at each $\mathcal{A}$. In both graphs the average $\left\langle C_{p}\right\rangle$ and $\mathcal{A}$ are presented for all the slits.

the functions. Figure 5(b) presents the conditional average of $\left\langle C_{p}\right\rangle$ at each $\mathcal{A}(t)$ for the filtered functions in all the slits. In both graphs, $\left\langle\overline{C_{p}}\right\rangle$ against $\overline{\mathcal{A}}$ are presented for all the slits.

As shown in Fig. 5, there is an instantaneous correlation between $\mathcal{A}$ and $\left\langle C_{p}\right\rangle$ for each slit size and a similar behavior is maintained across slit sizes. According to these figures, the main effect of the slit is to make the wake more symmetric, producing a base pressure increment as that expected in the baseline for an equivalent $\mathcal{A}$. Figure 5(b) provides further detail on the behavior of $\left\langle C_{p}\right\rangle$ at each $\mathcal{A}$. The unconverged ends, away from $\overline{\mathcal{A}}$, in each slit can be ignored. As observed, at low asymmetries, the $C_{p}$ of all the slits collapses with $\mathcal{A}$ and the pressure increment is mainly attained by improved wake symmetry. Nevertheless, at the highest values of $\mathcal{A}$, the largest slits provide further pressure drag reduction than that produced by improved wake symmetry. This is consistent with the hypothesis of diametrical cross flow across the cavity since, once $\mathcal{A}$ is fixed, the effect of the slit is expected to be larger for higher asymmetries. Also, the larger base pressure increase at large $\mathcal{A}$ can explain the reduction in $\left.\Delta \overline{\left\langle C_{p}\right.}\right\rangle / \Delta d$ at larger $d / D$ shown in Fig. 2(b). Extrapolating $\mathcal{A} \rightarrow 0$ leads to a maximum $\Delta\left\langle\overline{C_{p}}\right\rangle /\left\langle\overline{C_{p}}\right\rangle_{0} \sim 20 \%$, consistent with the results presented in [26].

A phenomenology compatible with this pressure behavior is sketched in Fig. 6: a statistically coherent toroidal vortex in the wake, like that observed in laminar flows in axisymmetric bodies [16] and in turbulent flows around the Ahmed body [32], is held responsible for the low- and high-pressure areas, affected by the vortex proximity to the base and by the jet induced by the vortex.

(a) baseline
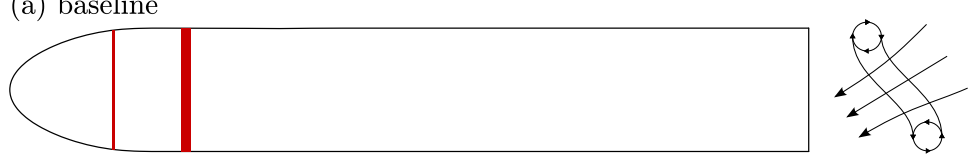

(b) slit - symmetrization

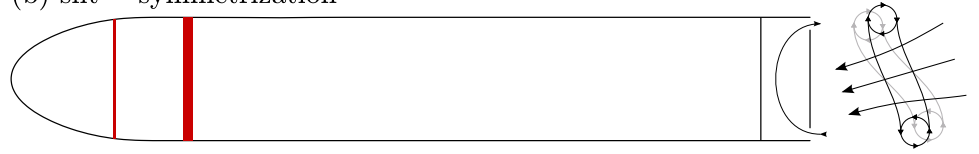

(c) slit - homogeneous $\Delta \mathrm{C}_{\mathrm{P}}$

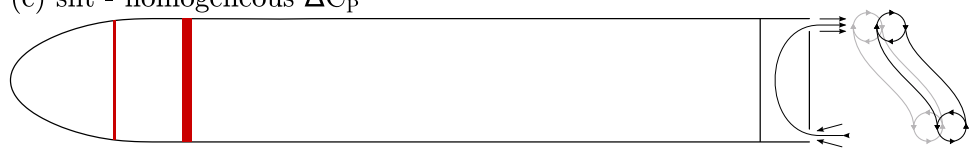

FIG. 6. Effect of the slit on the wake. (a) Mean asymmetric toroidal vortex in baseline. (b) Symmetrization of mean asymmetric toroidal vortex by slit. (c) Separation of mean asymmetric toroidal vortex by slit. 
In the scenario depicted in Fig. 6, the first-order effect of the slit is improved symmetry of the vortex position by reducing the pressure where it is highest and vice versa, with the associated streamed flow, as presented in Fig. 6(b). As a second-order effect, only noticeable at the highest values of $\mathcal{A}$, the velocity of the flow streamed out of the slit, where the vortex is closer, is more coherent than the velocity of the flow sucked into the cavity, where the vortex is farther away. As a result, the net effect over the vortex can be expected to increase its distance from the base, producing an overall pressure increment for the same values of $\mathcal{A}$ as those observed at high $\mathcal{A}$ in Fig. 5(b).

\section{CONCLUSIONS}

A novel passive mechanism producing base pressure increase on axisymmetric blunt bodies is presented. This consists of a thin perimetric slit located at the edge of the base of the body and communicating with the external flow via an internal cavity. A study of the effect of the cavity volume on the base pressure suggests that the mechanism relies on diametrical mass flow across the cavity. The main mechanism by which the slit increases the base pressure is observed to be an improved symmetry of the wake. As a secondary effect, the slit also increases the base pressure when the wake asymmetry is high. For the cases tested, the mean base pressure increases monotonically with slit size up to $\Delta\left\langle\overline{C_{p}}\right\rangle /\left\langle\overline{C_{p}}\right\rangle_{0} \sim 5 \%$ but the data indicate that a saturation at about $\Delta\left\langle\overline{C_{p}}\right\rangle /\left\langle\overline{C_{p}}\right\rangle_{0} \sim 20 \%$ should be expected.

The second effect of the slit is a global reduction of the pressure fluctuations in the base. Modal analysis of the pressure fluctuations shows that the slit affects mainly the random azimuthal rotation of the wake and the vortex shedding.

\section{ACKNOWLEDGMENTS}

We are indebted to EPSRC (EP/I005684/1) and the Imperial College EPSRC Impact Acceleration Account (EP/K503733/1) for financial support.

[1] J. B. Roberts, Coherence measurements in an axisymmetric wake, AIAA 11, 1569 (1973).

[2] H. V. Fuchs, E. Mercker, and U. Michel, Large-scale coherent structures in the wake of axisymmetric bodies, J. Fluid Mech. 93, 185 (1979).

[3] A. K. M. F. Hussain, Coherent structures - reality and myth, Phys. Fluids 26, 2816 (1983).

[4] H. J. Kim and P. A. Durbin, Observations of the frequencies in a sphere wake and of drag increase by acoustic excitation, Phys. Fluids 31, 3260 (1988).

[5] E. Berger, D. Scholz, and M. Schumm, Coherent vortex structures in the wake of a sphere and a circular disk at rest and under forced vibrations, J. Fluids Struct. 4, 231 (1990).

[6] G. Rigas, A. R. Oxlade, A. S. Morgans, and J. F. Morrison, Low-dimensional dynamics of a turbulent axisymmetric wake, J. Fluid Mech. 755, R5 (2014).

[7] H. Choi, W.-P. Jeon, and J. Kim, Control of flow over a bluff body, Annu. Rev. Fluid Mech. 40, 113 (2008).

[8] A. Roshko, On the drag and shedding frequency of two-dimensional bluff bodies, Tech. Rep. 3169 (NACA, 1954).

[9] P. W. Bearman, Investigation of the flow behind a two-dimensional model with a blunt trailing edge and fitted with splitter plates, J. Fluid Mech. 21, 241 (1965).

[10] P. W. Bearman, The effect of base bleed on the flow behind a two-dimensional model with a blunt trailing edge, Aero. Quart. 18, 207 (1967).

[11] M. Pastoor, L. Henning, B. Noack, R. King, and G. Tadmor, Feedback shear layer control for bluff body drag reduction, J. Fluid Mech. 608, 161 (2008).

[12] S. J. Lee and P. W. Bearman, An experimental investigation of the wake structure behind a disk, J. Fluids Struct. 6, 437 (1992). 
[13] Sadatoshi Taneda, Visual observations of the flow past a sphere at reynolds numbers between $10^{4}$ and $10^{6}$, J. Fluid Mech. 85, 187 (1978).

[14] A. R. Oxlade, High-frequency periodic jet forcing of a turbulent axisymmetric wake, Ph.D. thesis, Imperial College London, 2013.

[15] P. Bohórquez, E. Sanmiguel-Rojas, A. Sevilla, J. I. Jiménez-González, and C. Martínez-Bazán, Stability and dynamics of the laminar wake past a slender blunt-based axisymmetric body, J. Fluid Mech. 676, 110 (2011).

[16] Y. Bury and T. Jardin, Transition to chaos in the wake of an axisymmetric bluff body, Phys. Lett. A 376, 3219 (2012).

[17] V. Gentile, F. F. J. Schrijer, B. W. Van Oudeheusden, and F. Scarano, Time dependent behavior of the recirculation region behind an axisymmetric bluff body, in International Symposium on Turbulence and Shear Flow Phenomena (University of Melbourne, Melbourne, Australia, 2015).

[18] M. Grandemange, M. Gohlke, and O. Cadot, Reflectional symmetry breaking of the separated flow over three-dimensional bluff bodies, Phys. Rev. E 86, 035302 (2012).

[19] M. Grandemange, M. Gohlke, and O. Cadot, Turbulent wake past a three-dimensional blunt body. Part 1. Global modes and bi-stability, J. Fluid Mech. 722, 51 (2013).

[20] R. D. Brackston, J. M. García de la Cruz, A. Wynn, G. Rigas, and J. F. Morrison, Stochastic modelling and feedback control of bistability in a turbulent bluff body wake, J. Fluid Mech. 802, 726 (2016).

[21] J. L. F. Porteiro, C. E. G. Przirembel, and R. H. Page, Modification of subsonic wakes using boundary layer and base mass transfer, AIAA 21, 665 (1983).

[22] A. R. Oxlade, J. F. Morrison, A. Qubain, and G. Rigas, High-frequency forcing of a turbulent axisymmetric wake, J. Fluid Mech. 770, 305 (2015).

[23] A. Sevilla and C. Martínez-Bazán, Vortex shedding in high Reynolds number axisymmetric bluff-body wakes: Local linear instability and global bleed control, Phys. Fluids 16, 3460 (2004).

[24] W. A. Mair, The effect of a rear mounted disc on the drag of a blunt-based body of revolution, Aero. Quart. 16, 350 (1965).

[25] T. Morel, Effect of base cavities on the aerodynamic drag of an axisymmetric cylinder, Aero. Quart. 30, 400 (1979).

[26] P. R. Viswanath, Flow management techniques for base and afterbody drag reduction, Progress in Aerospace Sciences 32, 79 (1996).

[27] E. Sanmiguel-Rojas, J. I. Jiménez-González, P. Bohórquez, G. Pawlak, and C. Martínez-Bazán, Effect of base cavities on the stability of the wake behind slender blunt-based axisymmetric bodies, Phys. Fluids 23, 114103 (2011).

[28] N. Lin, H. L. Reed, and W. S. Saric, Instability, Transition, and Turbulence (Springer, New York, 1992), p. 421.

[29] F. M. White, Fluid Mechanics,7th ed. (McGraw-Hill, New York, 2011).

[30] G. Rigas, A. S. Morgans, R. D. Brackston, and J. F. Morrison, Diffusive dynamics and stochastic models of turbulent axisymmetric wakes, J. Fluid Mech. 778, R2 (2015).

[31] G. Vilaplana, M. Grandemange, M. Gohlke, and O. Cadot, Global mode of a sphere turbulent wake controlled by a small sphere, J. Fluids Struct. 41, 119 (2013).

[32] A. Evrard, O. Cadot, V. Herbert, D. Ricot, R. Vigneron, and J. Délery, Fluid force and symmetry breaking modes of a 3d bluff body with a base cavity, J. Fluids Struct. 61, 99 (2016). 